\title{
Variation for Resistance to Kernel Infection and Toxin Accumulation in Winter Wheat Infected with Fusarium graminearum
}

\author{
Clay Sneller, Mary Guttieri, Pierce Paul, José Costa, and Ryan Jackwood
}

First, second, and fifth authors: Department of Horticulture and Crop Science, The Ohio State University and the Ohio Agriculture Research and Development Center, 1680 Madison Ave, Wooster 44691; third author: Department of Plant Pathology, The Ohio State University and the Ohio Agriculture Research and Development Center; and fourth author: Department of Plant Science and Landscape Architecture, University of Maryland, 2102 Plant Sciences Bldg., College Park 20742-4452.

Accepted for publication 6 August 2011.

\begin{abstract}
Sneller, C., Guttieri, M., Paul, P., Costa, J., and Jackwood, R. 2012. Variation for resistance to kernel infection and toxin accumulation in winter wheat infected with Fusarium graminearum. Phytopathology 102:306-314.

Host resistance is the main way to control Fusarium head blight (FHB) in wheat. Despite improved levels of resistance to infection and spread in vegetative tissue, the toxin deoxynivalenol (DON) can still accumulate to unacceptable concentration levels. In this study, our objectives were to assess the genetic variation for resistance to kernel infection (RKI) and resistance to toxin accumulation (RTA) and their role in controlling DON. We collected spikes with different levels of visual symptoms from each of 32 wheat genotypes and at four environments and determined DON and fungal biomass (FB) from each sample. We assessed RKI by regressing FB on the level of visual symptoms and RTA by regressing DON on FB

for each genotype. Significant genetic effects were found for RKI and RTA. Some genotypes consistently had low FB in their grain despite increasing visual symptoms suggesting RKI. Additionally, some genotypes consistently had low DON in their grain despite increasing FB levels suggesting a higher RTA in these genotypes. The variation for RKI and RTA explained a significant fraction of the variation for DON among genotypes with moderate visual symptoms using independent grain samples. Although RKI and RTA were significantly correlated $(r=0.58$, $P=0.05)$, RTA was more predictive of DON accumulation because it modeled 32 to $44 \%$ of the genotype sum of squares for DON, while only 9 to $10 \%$ were predicted using RKI. Thus, variation for RTA was important in explaining variation for DON among genotypes with acceptable levels of resistance to fungal infection and spread. This work indicates that there is a need to develop a better understanding of RTA and rapid screening methods for this trait.
\end{abstract}

Fusarium head blight (FHB), caused by the fungus Fusarium graminearum Schwabe (teleomorph: Gibberella zeae (Schwein.) Petch), is a widespread and devastating disease of wheat (Triticum aestivum L.). The primary injury from FHB results from infection of flowering structures and grain and production of the trichothecene deoxynivalenol (DON). Accumulation of DON in the grain is an acute problem, because DON is toxic to humans and livestock $(26,34)$. Host resistance is the primary control measure for FHB. Mesterházy (28) postulated multiple mechanism of FHB resistance in small grains, including resistance to initial infection (type I resistance) and spread (type II resistance). These two mechanisms have been well documented in wheat and are used in breeding, because they are easy to assess visually. In addition, resistance to kernel infection (RKI) and resistance to toxin accumulation (RTA) were postulated (28). We have chosen to refer to these as RKI and RTA because, in the literature, RKI has been listed as type III $(27,28)$ and IV (29) while RTA has been listed as type V $(27,28)$ and type III $(29)$.

Both RTA and RKI are difficult to document because they require accurate measurement of kernel infection and are usually confounded by the effects of type I and II resistance on kernel

Corresponding author: C. Sneller; E-mail address: sneller.5@osu.edu

Any opinions, findings, conclusions, or recommendations expressed in this publication are those of the authors and do not necessarily reflect the view of the United States Department of Agriculture.

http://dx.doi.org/10.1094/PHYTO-05-11-0143

(C) 2012 The American Phytopathological Society infection and DON concentration. There are several reports that that some wheat genotypes had fewer Fusarium-damaged kernels (FDK) (a visual estimate of the percentage of infected kernels) or less DON than expected based on their type I and II resistance levels $(29,41)$ but these studies did not repeat these deviations, and actual infection levels were not determined. Few studies have documented the variation in RKI or RTA $(10,35,39)$, although both could be very useful in reducing the DON concentration of grain and breeding for resistance.

The traits used in selecting for FHB resistance are often related when assessed over a wide range of resistance levels $(31,32)$. Selection for type I and II resistance expressed as visual symptoms reduces the proportion of FDK from a spike and lowers DON levels. Many mapping studies have reported that quantitative trait loci (QTL) for type I or II resistance also affect FDK or DON in the grain $(5,23,39)$. The chromosome region with the most important QTL for type II resistance ( $Q f h s-n d s u-3 B S)$ has been associated with the ability to detoxify DON (22), and several studies have indicated that wheat spikes from cultivars with type II resistance degrade DON more than susceptible cultivars $(22,30,47)$.

Some researchers have reported QTL for FDK or DON that are not associated with type I or II resistance $(14,17,20,24,38,42,48)$, suggesting that these are genes for RKI or RTA. However, these studies did not directly assess kernel infection and the QTL have seldom been validated. In addition, most mapping studies use small populations that segregate for genes affecting type I and II resistance, leading to confounding measurements of RKI or RTA from simply assessing FDK or DON. Thus, these FDK-only and DON-only QTL could result from many factors other than true RKI or RTA. One clear exception is a validated QTL that only 
affects DON level in barley (39) that must result from RKI or RTA.

The association of grain DON concentration and visual symptoms of FHB is weak among cultivars with good type I or II resistance, and grain from the moderately resistant cultivars can still have unacceptable DON levels (31-33). Healthy looking kernels may actually be infected (7) and contain DON $(6,7)$. There is some evidence that type II resistance can lead to increased DON concentration in grain from infected florets (44). It is apparent that type I and II resistance must be complemented with RKI and RTA to reliably attain low concentration of DON in grain.

Direct selection for RKI or RTA would be a fundamental shift in breeding for FHB resistance. For RKI, one needs to show that the grain of a genotype repeatedly has less kernel infection than expected based on visual symptoms in vegetative tissue. Most breeders use FDK to assess kernel infection. This is convenient but is not a precise measure of kernel infection $(6,7)$. In order to assess RTA, one needs to show that the grain of a genotype repeatedly has lower DON than expected based on the degree of kernel infection. In other words, in order to adequately select for RTA and RKI, the confounding effects of type I and II resistance on kernel infection and DON contamination of grain need to be minimized. There is a need to confirm genetic variation for RKI and RTA and determine their importance in controlling DON levels in grain. In this study, our objectives were to (i) assess RKI and RTA in a set of wheat genotypes and (ii) determine whether variation for RKI and RTA explained variation in DON content in a set of wheat genotypes with good type I and II resistance.

\section{MATERIALS AND METHODS}

Description of trials. In 2006, 2007, and 2008, 75 recombinant inbred lines (RIL), checks, and some parents of the RILs (Table 1) were grown in FHB nurseries in Wooster, $\mathrm{OH}$ on the Snyder farm of the Ohio Agricultural Research and Development Center (all years) and in Salisbury, MD in 2008. Each trial consisted of a randomized complete block design with three replications in Ohio and two in Maryland. Each replication was a single 0.91-m row with $0.30 \mathrm{~cm}$ between individual rows. Planting dates varied between 25 September and 15 October. The nurseries were fertilized according to soil test recommendations and supplied with $\mathrm{N}$ at $22 \mathrm{~kg} / \mathrm{ha}$ in the fall and $78 \mathrm{~kg} / \mathrm{ha}$ in the spring between Feekes growth stages 4 and 6 . Weeds were controlled with standard herbicides.

The Ohio locations were inoculated with maize grain spawn as described by Sneller et al. (40). The field was mist irrigated intermittently for 2 min every 10 min between 0500 and $0900 \mathrm{~h}$ and between 2000 and $2200 \mathrm{~h}$ immediately after broadcasting the inoculum and every day until 3 days after the latest line reached anthesis. In Maryland, scabby corn inoculum was applied $\approx 30$ days before flowering as described by Kang et al. (18), and misting was applied daily for $2 \mathrm{~h}$ in the morning (0600 to $0800 \mathrm{~h}$ ) and $2 \mathrm{~h}$ in the evening (1900 to $2100 \mathrm{~h}$ ) until physiological maturity.

We measured the FHB index (IND) and heading dates (HD; Feekes stage 10.5) for all genotypes from each plot. IND on a whole plot was determined by visually estimating the percentage of symptomatic spikelets in three locations in a plot. HD was determined as the day when $50 \%$ of the spikes in a plot had fully emerged from the boot (i.e., the leaf sheath of the flag leaf). Grain was harvested from each replication of each genotype in each year and threshed. The grain from all replications of a genotyped was bulked for DON analysis. DON analyses were performed at the U.S. Wheat and Barley Scab Initiative-funded DON testing laboratory at the University of Minnesota laboratory using gas chromatography-mass spectrometry (12).

Sampling for RKI and RTA. In 2007 in Wooster, we tagged spikes with different numbers of symptomatic spikelets (SS) in each replication of each of 75 genotypes. This trial was an initial screen for variation for RKI and RTA. At 21 days after anthesis, spikes with $0,1,2$, or $3 \mathrm{SS}$ were tagged with different colors. We focused on low levels of infection to mimic infection levels likely to occur in moderately resistant germplasm. Tagging five spikes of each category was attempted. This was not always possible, although at least one spike of each type was tagged in each plot. At maturity, spikes with different SS levels of each genotype were harvested from each plot and bulked. Thus, for each of the 75 genotypes in 2007, we created bulks of spikes with $0,1,2$, and $3 \mathrm{SS}$, for a total for 300 samples.

This was repeated in 2008 on only 32 genotypes. These 32 genotypes were selected as the most resistant and susceptible, based on results from the 2007 experiments. In 2008, we tagged

TABLE 1. Pedigree and number of recombinant inbred lines (RILs) and names of the checks and parents tested from 2006 to 2008

\begin{tabular}{|c|c|c|}
\hline Pedigree or name & Number of RILs & Number of RILs also assayed for $\mathrm{FB}^{\mathrm{a}}$ \\
\hline Hopewell/Ernie & 10 & $5(2-1,2-2,2-3,2-4,2-5)$ \\
\hline Hopewell/OH599 & 9 & $4(1-1,1-2,1-3,1-4)$ \\
\hline OH552/Hopewell & 9 & $3(3-1,3-2,3-3)$ \\
\hline Hopewell/P.92227C5-1-1 & 4 & $3(6-1,6-2,6-3)$ \\
\hline OH631/Hopewell & 4 & $2(5-1,5-2)$ \\
\hline OH626/Hopewell & 4 & $2(10-1,10-2)$ \\
\hline Hopewell/OH655 & 4 & \\
\hline A93-6227/Hopewell & 2 & $1(4-1)$ \\
\hline OH645/OH561 & 3 & $2(7-1,7-2)$ \\
\hline Hopewell/BL930390 & 2 & $2(13-1,13-2)$ \\
\hline Hopewell/IL94-6858 & 2 & $1(12-1)$ \\
\hline OH560/Hopewell & 1 & $\ldots$ \\
\hline Hopewell/OH536 & 2 & $\ldots$ \\
\hline Hopewell/IL94-6734 & 1 & $\ldots$ \\
\hline Freedom & Check & $\ldots$ \\
\hline Pioneer 2545 & Check & $\ldots$ \\
\hline Truman & Check & 1 (TRUM) \\
\hline
\end{tabular}

a Number of RILs also assayed for fungal biomass (FB) in 2008 and their codes used in Table 4. 
eight spikes of each SS level per plot of each genotype. The spikes of a given SS level from each plot of each genotype were hand harvested and bulked separately to form one sample. Thus, in 2008, we had 12 samples from each genotype (0 SS from replication 1, 0 SS from replication $2,0 \mathrm{SS}$ from replication $3, \ldots$, 3 SS from replication 3). This resulted in a total of 384 samples in 2008. Each sample was carefully threshed to minimize loss of grains. We assessed DON and fungal biomass (FB) of each sample.

Processing RKI and RTA samples. Individual grain samples were ground with a Falling Number grinder (Perten Instruments, Stockholm), which was cleaned between each sample. A 1-g sample of ground grain was weighed into a plastic twist-top bag and sent for DON analysis as described above. A 0.1-g sample of ground grain was weighed into a $1.5-\mathrm{ml}$ microcentrifuge tube for DNA extraction.

DNA was extracted using the DNeasy 96 Plant Kit (Qiagen Group, Valencia, CA). A 0.4-ml aliquot of lysis buffer AP1 was added to the grain sample in the microcentrifuge tube; then, $2 \mu \mathrm{l}$ of RNAse was added and the pipette tip used to mix the grain sample with the lysis buffer. The sample was vortexed well, then heated at $65^{\circ} \mathrm{C}$ for $10 \mathrm{~min}$ with occasional mixing. Following the manufacturer's directions, $130 \mu \mathrm{l}$ of solution AP2 was added and the sample was mixed well and placed on ice for $5 \mathrm{~min}$. Samples were centrifuged at $20,000 \times g$ for $5 \mathrm{~min}$. A 400- $\mu \mathrm{l}$ aliquot of each supernatant was transferred to a 1.2-ml deep-well tube in a 96-well array, and the extractions proceeded as described by the manufacturer in the DNeasy Plant Handbook (07/2006). DNA concentrations were determined using a NanoDrop (NanoDrop Products, Wilmington, DE). Concentrations were normalized to $10 \mathrm{ng} \mu \mathrm{l}^{-1}$. DNA samples then were diluted 1:1 with Tris-EDTA buffer (10 mM Tris-HCl, $\mathrm{pH} 8.0$, and $1 \mathrm{mM}$ EDTA) to a final concentration of $5 \mathrm{ng} \mathrm{\mu l}^{-1}$ for real-time polymerase chain reaction (RT-PCR).

RT-PCRs were conducted in triplicate with a standard curve in each plate. Reactions contained $2.5 \mu$ of DNA solution and 17.5 $\mu \mathrm{l}$ of enzyme-primer solution. The final reaction was $1 \times$ PerfeCta Multiplex qPCR SuperMix (Quanta BioSciences, Gaithersburg, MD) and contained $500 \mathrm{nM}$ each unlabeled primer and $250 \mathrm{nM}$ each labeled probe. The PCR conditions included an initial 7-min denaturation at $95^{\circ} \mathrm{C}$ followed by 45 cycles of a 15-s denaturation at $95^{\circ} \mathrm{C}$ and $1 \mathrm{~min}$ of annealing/extension at $60^{\circ} \mathrm{C}$. Primers and probe for $F$. graminearum DNA quantification were as described by Waalwijk et al. (45). Primers and probe for T. aestivum DNA quantification were based on Puroindoline B sequences (PinB-F, 5'-TGGCGGAGGAGGTGGTTC-3'; PinB-R, 5'-CGACATTGT GGTGCTATCTGG-3'; and probe, HEX-aaatggtggaagggcggctgTAMRA).

Detection of both FAM and HEX chromophores was conducted during the annealing/extension step. Data collected during the last $75 \%$ of the annealing/extension step time were used for analysis. Threshold cycles were set to provide optimal separation of standard curve data.

Statistics. Set of 32 genotypes assessed for DON and FB in 2007 and 2008. The ratio of $F$. graminearum DNA to wheat DNA $(\mu \mathrm{g} / \mu \mathrm{g})$ was used to estimate FB. These ratio data showed significant skewness and kurtosis; therefore, the log of the ratio was used in all analyses. There was one set of samples per genotype per SS level in 2007 (pooled over three replications) and three sets of samples in 2008 (one from each replication). In the analysis, we used levels of set to classify samples instead of a combination of year and replication levels due to the fact that we had just one replication in 2007 and three in 2008. We conducted an analysis of variance (ANOVA) considering set as a random effect and genotype and SS fixed effects.

To assess RKI of the 32 genotypes, we regressed FB on SS level (levels $0,1,2$, and 3 ) in each set of samples for each genotype to obtain four estimates of the slope per genotype. In this analysis, SS serves as an estimate of the degree of spike infection: genotypes with good RKI should have low slopes while those with poor RKI should have steeper positive slopes. We conducted an ANOVA of the slopes using a completely randomized design to assess the significance of genotype and set effects on the slope. We also conducted a regression for each genotype using all 16 data points (four SS levels by four sets) per genotype to obtain a single estimate of slope for each genotype.

To assess RTA of the 32 genotypes, we regressed DON on FB obtained from each SS level in each set for each genotype to obtain four estimates of the slope per genotype. In this analysis, the levels of SS among the samples within a set simply provided variation for FB. Genotypes with good RTA should have low slopes while those with poor RTA should have steeper positive slopes. We conducted an ANOVA of the slopes using a completely randomized design to assess the significance of genotype and set effects on slope. We also conducted a regression using all 16 data points (four SS levels by four sets) to obtain a single estimate of slope for each genotype.

Set of 75 genotypes assessed for IND and DON. We performed an ANOVA for the IND and DON data from the 75 genotypes assessed in 2006, 2007, and 2008 in Ohio and in 2008 in Maryland. The IND and DON data for this analysis were collected on whole plots rather than individual spikes. In Ohio, we pooled grain over replications prior to DON analysis; therefore, we only had one DON observation per genotype per environment. For Ohio, we averaged the IND values for a genotype over the three reps from a year to obtain one IND observation per genotype that corresponded to the single DON observation. In the Maryland trial, we obtained DON and IND from each of two replications. The values from each plot of each genotype were averaged and used as a single observation from that environment.

Environments and genotypes were considered random in the analysis because the genotypes were a random selection of moderately resistant lines. Entry mean heritability and its error were calculated (19). We regressed DON on IND and obtained the residual for each genotype. Two regression analyses were done (i) for each of the four environments, thus producing four residuals per genotype, and (ii) one regression using the mean IND and DON values over all environments, producing one residual per genotype.

We conducted an ANOVA of DON per se and of the residuals from analyses (i) above using a completely randomized design to assess the significance of genotype and environment effects. This ANOVA was repeated using just the data from the 32 genotypes assessed for RKI and RTA: these analyses were conducted with and without IND, HD, and the estimates of RKI and RTA as covariates. Multiple covariates were entered in the model in order of their importance in the single covariate analyses. The covariate analyses were repeated using data just from Maryland in 2008 and Ohio 2006 because these environments were not sampled to determined RKI and RTA.

All ANOVA, correlation, and regression analyses were conducted using SAS software (36).

\section{RESULTS}

The RILs in this study were selected because they had moderate to low IND values. Theses RILs were not very diverse because $41 \%$ of the parentage comes from The Ohio State University (OSU) cultivar Hopewell, 33\% comes from other OSU breeding lines, $12 \%$ from Purdue University breeding lines, $8 \%$ from 'Ernie', 4\% from University of Illinois breeding lines, and 3\% from other two other sources (Table 1).

Estimating genetic effects for RKI and RTA. The ANOVAs of DON and FB from the 32 genotypes tested in four sets of samples in 2007 and 2008 produced similar results for both traits (Table 2). All effects were significant $(P<0.05)$, except for the 
set-SS interaction. Both DON and FB increased as SS increased (Table 3). The three sets sampled from 2008 were similar to one another and all had more DON and FB than the one sampled from 2007 (Table 3).

TABLE 2. Analysis of variance for deoxynivalenol (DON) and fungal biomass (FB) of grain sampled from spikes with different levels of symptomatic spikelets (SS) from four sets of samples collected from 2007 (one set) and 2008 (three sets) trial of 32 wheat genotypes

\begin{tabular}{lrcc}
\hline & & \multicolumn{2}{c}{$F$ test $^{\mathrm{a}}$} \\
\cline { 3 - 4 } Source & df & DON & FB \\
\hline Set & 3 & $77.1^{* *}$ & $43.6^{* *}$ \\
SS & 3 & $132.9^{* *}$ & $24.2^{* *}$ \\
SS $\times$ set & 9 & 1.19 & 1.64 \\
Genotype & 31 & $5.48^{* *}$ & $3.23^{* *}$ \\
Genotype $\times$ set & 93 & $2.54^{* *}$ & 1.35 \\
Genotype $\times$ SS & 93 & $3.41^{* *}$ & $2.16^{* *}$ \\
Error & 281 & $\ldots$ & $\ldots$
\end{tabular}

a Asterisks: *,**, and $* * *$ indicate significance at $P$ levels $<0.05,0.01$, and 0.001 , respectively.
Within each set of samples, we regressed FB on SS and the slope was used as the estimate of RKI. Within each set, we regressed DON on FB and the slope was used as the estimate of RTA. There were a total of 128 regressions (four sets by 32 genotypes) estimating RKI and 128 regressions estimating RTA. For RKI, 15 of 128 individual regressions were significant, while 22 of 128 were significant for RTA. The number of significant regressions was fairly uniformly distributed across the four sets of samples. The low percentage of significant regressions is not surprising because each individual analysis had just 2 degrees of freedom in the error term. In addition, genotypes with good resistance should have a flat slope that would not model much variation: this would be a desired outcome. When all 16 data points per genotype (four levels of SS by four sets) were included in a single analysis, then 16 of 32 regressions were significant for RKI and 19 of 32 were significant for RTA (Table 4).

An ANOVA was conducted for the estimates of slope for RKI and RTA obtained from the four sets of samples. Genotype effects were significant for RKI and RTA slopes while set effects were not (Table 5). The genotype effects accounted for 33 and $37 \%$ of

TABLE 3. Average deoxynivalenol (DON) and fungal biomass (FB) by number of symptomatic spikelets (SS) and environment from the four sets of samples obtained from 2007 and 2008 trials of 32 wheat genotypes ( $\mathrm{SD}=$ standard deviation)

\begin{tabular}{lccccc}
\hline Number of SS & DON $(\mu \mathrm{g} / \mathrm{kg})(\mathrm{SD})$ & FB $(\mu \mathrm{g})(\mathrm{SD})$ & Sample set $($ year $)$ & DON $(\mu \mathrm{g} / \mathrm{kg})(\mathrm{SD})$ & FB $(\mu \mathrm{g})(\mathrm{SD})$ \\
\hline 0 & $2.9(2.3)$ & $0.19(1.22)$ & $1(2008)$ & $6.6(5.3)$ & $1.24(1.22)$ \\
1 & $6.1(4.2)$ & $1.03(1.12)$ & $2(2008)$ & $7.2(4.9)$ & $1.08(1.12)$ \\
2 & $7.4(5.2)$ & $1.10(1.31)$ & $3(2008)$ & $3.9(4.8)$ & $1.21(1.05)$ \\
3 & $9.1(5.4)$ & $1.30(1.17)$ & $4(2007)$ & $3.8)$ & $0.15(1.38)$ \\
\hline
\end{tabular}

TABLE 4. Intercept (Int) and slope $(b)$ from assessing resistance to kernel infection (RKI) by regressing fungal biomass (FB) of kernels on number of symptomatic spikelets (SS) and resistance to toxin accumulation (RTA) by regressing grain deoxynivalenol (DON) on kernel FB of 32 wheat genotypes tested in 2007 and $2008^{\mathrm{a}}$

\begin{tabular}{|c|c|c|c|c|c|c|c|c|}
\hline \multirow[b]{2}{*}{ Genotype code } & \multicolumn{4}{|c|}{ RKI from regressing FB on SS } & \multicolumn{4}{|c|}{ RTA from regressing DON on FB } \\
\hline & Int & $b$ & $r^{2}$ & Class & Int & $b$ & $r^{2}$ & Class \\
\hline $1-1$ & 0.40 & 0.38 & 0.23 & M & 3.86 & 2.96 & $0.44 *$ & Var \\
\hline $1-2$ & -0.58 & 0.87 & 0.36 & MS & 5.61 & 2.64 & $0.45^{*}$ & Var \\
\hline $1-4$ & 0.01 & 0.25 & 0.11 & Var & 5.22 & 3.47 & 0.30 & Var \\
\hline $2-1$ & -1.00 & 0.00 & 0.00 & MR & 3.85 & 0.51 & 0.05 & Var \\
\hline $2-2$ & -0.30 & 0.75 & 0.37 & $\mathrm{~S}$ & 5.46 & 1.11 & 0.26 & Var \\
\hline $3-1$ & 0.60 & 0.46 & $0.44 *$ & M & 2.61 & 3.80 & $0.63 * *$ & MS \\
\hline $3-2$ & -0.01 & 0.52 & 0.37 & M & 3.48 & 2.28 & 0.33 & M \\
\hline $3-3$ & 0.45 & 0.42 & 0.26 & M & 1.66 & 4.34 & $0.71 * *$ & MS \\
\hline $4-1$ & 0.56 & 0.44 & 0.27 & Var & 1.67 & 2.82 & $0.68^{* * *}$ & Var \\
\hline $5-1$ & -0.58 & 0.67 & $0.60 * *$ & MS & 5.75 & 3.06 & $0.42 *$ & MS \\
\hline $5-2$ & 0.10 & 0.37 & 0.32 & Var & 4.19 & 2.22 & $0.68 * *$ & Var \\
\hline $8-1$ & 0.29 & 0.37 & 0.10 & M & 3.12 & 2.17 & $0.62 * *$ & M \\
\hline $8-2$ & 0.79 & 0.50 & $0.72 * *$ & M & 2.56 & 7.27 & $0.46^{*}$ & $\mathrm{~S}$ \\
\hline $9-1$ & 0.78 & -0.08 & 0.00 & MR & 2.78 & 0.67 & 0.18 & $\mathrm{R}$ \\
\hline $9-2$ & 0.72 & -0.05 & 0.00 & Var & 2.86 & 0.86 & 0.09 & Var \\
\hline $10-1$ & 1.61 & 0.37 & 0.32 & M & 1.26 & 1.90 & $0.53 *$ & M \\
\hline $10-2$ & 0.97 & 0.62 & 0.30 & MS & 0.83 & 1.27 & $0.52 *$ & M \\
\hline $12-1$ & 1.16 & 0.34 & 0.09 & Var & 6.68 & 0.53 & 0.03 & Var \\
\hline $13-1$ & 0.93 & 0.31 & 0.24 & Var & 4.87 & -0.90 & 0.06 & $\mathrm{R}$ \\
\hline $13-2$ & 0.96 & -0.13 & 0.01 & Var & 4.27 & 0.16 & 0.01 & $\mathrm{R}$ \\
\hline $14-1$ & 0.33 & 0.48 & 0.29 & Var & 3.37 & 2.36 & 0.39 & $\mathrm{M}$ \\
\hline HOPE & 0.44 & 0.32 & 0.21 & M & 4.08 & 3.08 & $0.51 *$ & MS \\
\hline TRUM & 0.77 & 0.20 & 0.01 & Var & 5.10 & 0.74 & 0.21 & MS \\
\hline $\mathrm{LSD}^{\mathrm{b}}$ & $\ldots$ & 0.64 & $\ldots$ & $\ldots$ & $\ldots$ & 5.54 & $\ldots$ & $\ldots$ \\
\hline
\end{tabular}

a Both regressions used all data from all 16 grain samples (four levels of SS by four sets). For $r^{2}$ probability, *, **, and $* * *$ indicate significance at $P$ levels $<0.05$, 0.01 , and 0.001 , respectively. For class, susceptible (S), moderately susceptible (MS), moderate (M), moderately resistant (MR), and resistant [R], and variable (Var) ratings were determined by assessing the consistency of the estimates of slope from each of the four sets of samples.

b Least significant difference. 


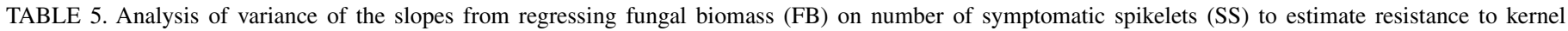
infection (RKI) and from regressing deoxynivalenol (DON) on FB to estimate resistance to toxin accumulation (RTA) ${ }^{\mathrm{a}}$

\begin{tabular}{|c|c|c|c|c|c|c|c|}
\hline \multirow[b]{2}{*}{ Source } & \multirow[b]{2}{*}{ df } & \multicolumn{3}{|c|}{ Slopes from regressing FB on SS to estimate RKI } & \multicolumn{3}{|c|}{ Slopes from regressing DON on FB to estimate RTA } \\
\hline & & Sum of squares & Mean square & $F$ test & Sum of squares & Mean square & $F$ test \\
\hline Set & 3 & 1.4 & 0.48 & 2.3 & 40 & 13.2 & 0.9 \\
\hline Genotype & 31 & 10.3 & 0.33 & $1.6^{*}$ & 895 & 28.9 & $1.9 *$ \\
\hline Error & 93 & 19.4 & 0.21 & $\ldots$ & 1,449 & 15.6 & $\ldots$ \\
\hline
\end{tabular}

a Slopes were obtained from 32 genotypes from a total four sets of samples obtained from 2007 and $2008 ; *$ indicates significance at $P$ levels $<0.05$

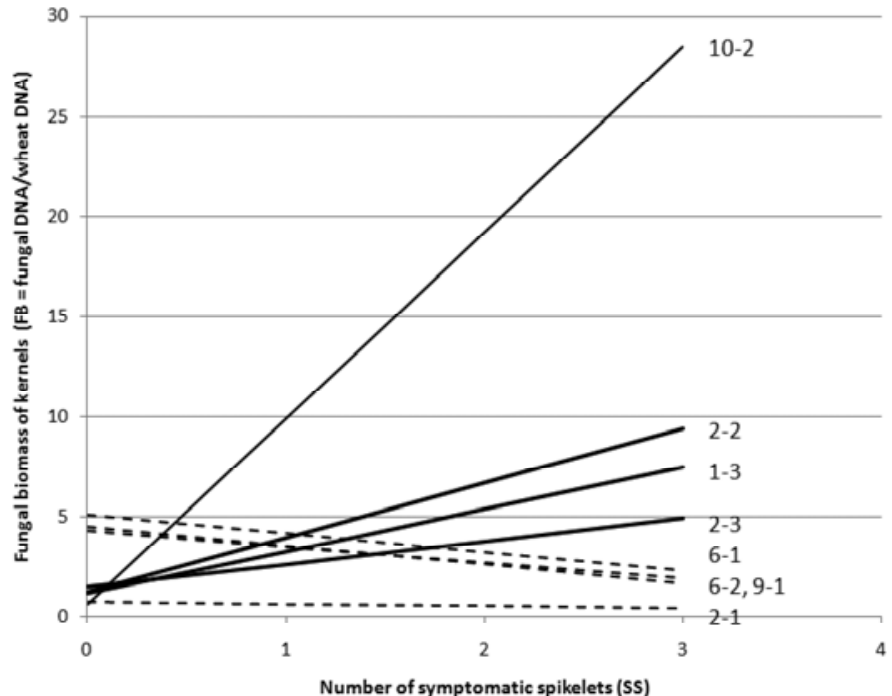

Fig. 1. Predicted response of fungal infection of kernels to increasing number of symptomatic spikelets (SS) of three lines with good resistance to kernel infection (RKI, dashed lines) and three with poor RKI (solid lines). Fungal infection was estimated by fungal biomass (FB) using nontransformed data.

the total sum of squares (SoS) for RKI and RTA, respectively. The four estimates of slopes from the four sets of samples for each genotype were visually assessed to determine whether some genotypes had slopes that were consistently above, below, or near the average slope of a set.

For RKI, 10 genotypes had variable slopes (e.g., sometimes above average and sometimes below average), 10 were consistently moderate (e.g., close to the average in all environments), 8 showed consistent evidence of susceptibility (e.g., slopes greater than average), and 4 showed consistent evidence of resistance (Table 4). For example, genotype 2-2 had the greatest slope $(b=$ 0.75 ) when all 16 data points were used in the regression (Table 4 ); its slope in the four sets was $1.06,0.36,0.85$, and 0.75 , which were all greater than the average slope in each set. Genotype 6-2 had a slope of -0.35 overall and slopes of $-0.47,-0.54,-0.51$, and 0.14 (still below average) in the four sets.

For RTA, 13 genotypes had variable slope, 6 were consistently moderate, 7 showed consistent evidence of susceptibility, and 5 showed consistent evidence of resistance (Table 4). For example, genotype 8-2 had the second greatest slope $(b=7.27)$ when all 16 data points were used in the regression (Table 4): its slope in the four sets was 5.6, 6.5, 10.1, and 6.0, which were all greater than the average slope in each set. Genotype 13-1 had a slope of -0.90 overall and slopes of $-0.1,-2.0,-0.3$, and -2.2 in the four sets. The slopes for RKI and RTA of the 32 genotypes estimated using all data on regressing were significantly correlated $(r=0.58)$.

Slopes of the best and worst genotypes for RKI are shown in Figure 1, while Figure 2 shows the slope of the best and worst for RTA. The genotypes with the best RKI showed little increase in FB despite increasing level of visual symptoms on the spike. The genotypes with the best RTA showed little increase in DON despite relatively high FB levels in the grain.

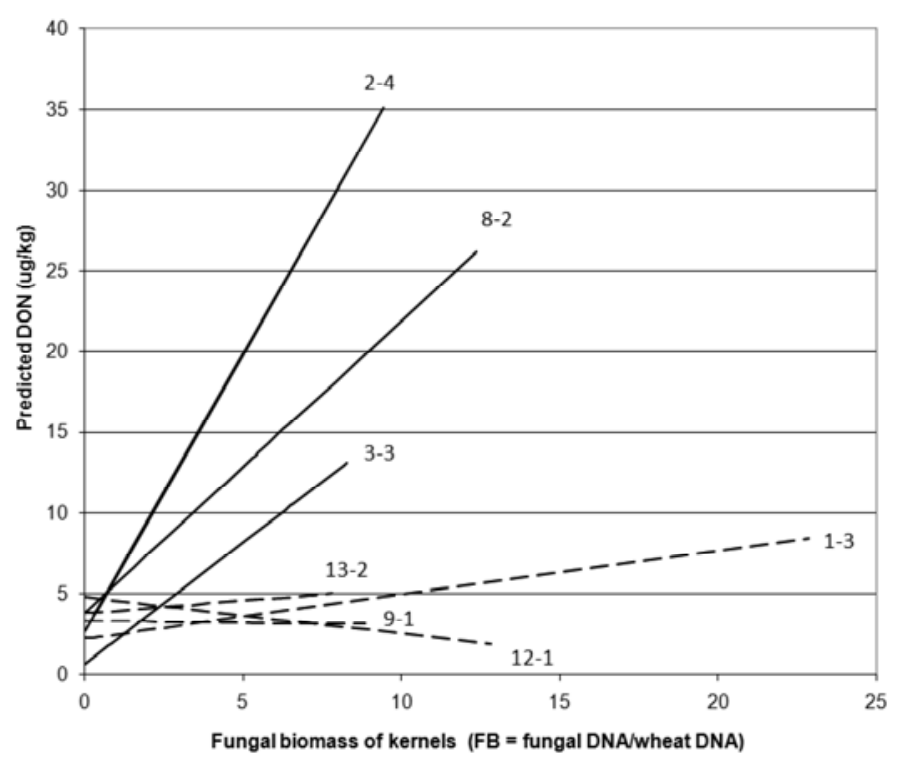

Fig. 2. Predicted response of grain deoxynivalenol (DON) levels to increasing fungal kernel infection of four lines with good resistance to toxin accumulation (RTA, dashed lines) and three with poor RTA (solid lines). Fungal infection is estimated by fungal biomass (FB) using nontransformed data.

Association of RKI and RTA with DON and IND data from whole plots. In all, 75 wheat genotypes were evaluated for IND and DON on a whole-plot level in four environments, including the 32 genotypes that were assessed for RKI and RTA. The ANOVA for IND and DON indicated significant effects of environment and genotype (Table 6). Over the four environments, heritability was 0.68 (95\% confidence intervals: 0.57 and 0.73 ) for IND and 0.49 (95\% confidence intervals: 0.30 and 0.57 ) for DON. All RILs were moderately resistant because all had mean IND values less than that of the susceptible ('Pioneer 2545', IND $=28.8$ ) and the moderate (Hopewell, IND = 18.8) check. Twenty-one RILs had mean DON levels greater than that of Pioneer $2545(4.5 \mu \mathrm{g} / \mathrm{kg})$.

The regression of DON on IND was significant in two of four environments and in the regression that used genotype means over all environments (Table 7). The $r^{2}$ values were modest even when the regression was significant. This result was not surprising because we had selected genotypes with moderate to low IND and the relationship of DON to IND may be nonsignificant among such genotypes, with the variation in IND explaining only a very small percent of the variation in DON (33). We also collected HD data from each environment. The correlation of HD and IND was significant in MD in $2008(r=-0.50)$ but not in the OH environments. The correlation of HD with DON was significant only in OH $2006(r=0.36)$.

We conducted an ANOVA of the deviation of observed DON from predicted DON (e.g., the residuals) of each genotype from each environment using data from all 75 genotypes and then using data from just the 32 genotypes with estimates of RKI and RTA (Table 8). Similar results were obtained in both analyses, with genotype effects being highly significant (Table 9). This was expected given the mostly nonsignificant regressions and low 
TABLE 6. Analysis of variance for index (IND) and deoxynivalenol (DON) for 75 wheat genotypes tested in four environments in 2006,2007 , and $2008^{a}$

\begin{tabular}{|c|c|c|c|c|c|c|c|}
\hline \multirow[b]{2}{*}{ Source } & \multirow[b]{2}{*}{ df } & \multicolumn{3}{|c|}{ IND } & \multicolumn{3}{|c|}{ DON } \\
\hline & & Sum of squares & Mean square & $F$ test & Sum of squares & Mean square & $F$ test \\
\hline Environment & 3 & 9,324 & 3,108 & $118.8 * * *$ & 1,367 & 456.6 & $76.2 * * *$ \\
\hline Genotype & 74 & 6,080 & 77 & $2.9 * * *$ & 862 & 10.9 & $1.8 * * *$ \\
\hline Error & 222 & 5,362 & 26 & $\ldots$ & 1,219 & 6.0 & $\ldots$ \\
\hline
\end{tabular}

a $* * *$ indicate significance at $P$ levels $<0.001$.

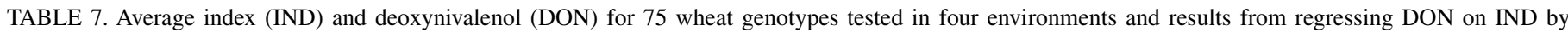
environment and from an analysis using genotype means over environments

\begin{tabular}{|c|c|c|c|c|c|c|}
\hline \multirow[b]{2}{*}{ Environment } & \multicolumn{2}{|c|}{ Average } & \multicolumn{4}{|c|}{ Regression } \\
\hline & IND $(\%)$ & $\mathrm{DON}(\mu \mathrm{g} / \mathrm{kg})$ & Intercept & Slope & Probability & $r^{2}$ \\
\hline $2006 \mathrm{OH}$ & 10.1 & 8.2 & 6.24 & 0.24 & 0.159 & 0.04 \\
\hline $2007 \mathrm{OH}$ & 11.0 & 2.2 & 0.26 & 0.18 & $<0.001$ & 0.34 \\
\hline $2008 \mathrm{OH}$ & 19.9 & 3.3 & 1.47 & 0.09 & $<0.001$ & 0.24 \\
\hline $2008 \mathrm{MD}$ & 4.1 & 3.4 & 3.33 & 0.02 & 0.839 & 0.00 \\
\hline Genotype means & 9.7 & 3.9 & 2.93 & 0.10 & 0.048 & 0.05 \\
\hline
\end{tabular}

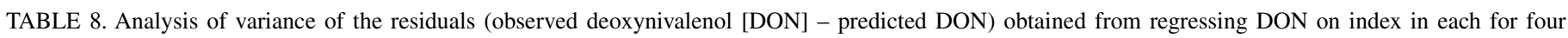
environments from 2006 to $2008^{\mathrm{a}}$

\begin{tabular}{|c|c|c|c|c|c|c|c|c|}
\hline \multirow[b]{2}{*}{ Source } & \multicolumn{4}{|c|}{75 genotypes } & \multicolumn{4}{|c|}{32 genotype } \\
\hline & df & Sum of squares & Mean square & $F$ test & df & Sum of squares & Mean square & $F$ test \\
\hline Environment & 3 & 130 & 43.3 & $6.6 * * *$ & 3 & 70 & 23.3 & $3.1^{*}$ \\
\hline Genotype & 74 & 920 & 12.4 & $1.9 * * *$ & 31 & 553 & 17.8 & $2.4 * * *$ \\
\hline Error & 222 & 1,452 & 6.5 & $\ldots$ & 93 & 694 & 7.5 & $\ldots$ \\
\hline
\end{tabular}

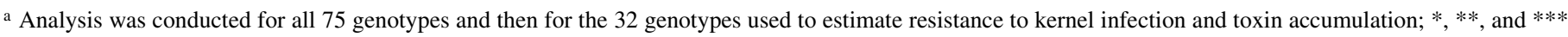
indicate significance at $P$ levels $<0.05,0.01$, and 0.001 , respectively.

TABLE 9. Sum of squares, expressed as a percentage of the total sum of squares, from the analysis of deoxynivalenol (DON) from 2006,2007 , and $2008^{a}$

\begin{tabular}{|c|c|c|c|c|c|c|c|c|c|c|c|c|c|c|}
\hline \multirow[b]{2}{*}{ Covariate } & \multicolumn{7}{|c|}{ All environments } & \multicolumn{7}{|c|}{ Two environments } \\
\hline & Env & Geno & Error & RTA & RKI & IND & HD & Env & Geno & Error & RTA & RKI & IND & HD \\
\hline None & $35.8 * * *$ & $27.2 * * *$ & 36.9 & $\ldots$ & $\ldots$ & $\ldots$ & $\ldots$ & $30.1 * * *$ & $53.4 * *$ & 16.5 & $\ldots$ & $\ldots$ & $\ldots$ & $\ldots$ \\
\hline RTA & $36.6 * * *$ & 18.6 & 36.9 & $7.9 * * *$ & $\ldots$ & $\ldots$ & $\ldots$ & $31.5 * * *$ & 34.0 & 16.5 & $18.0 * * *$ & $\ldots$ & $\ldots$ & $\ldots$ \\
\hline RKI & $35.2 * * *$ & $24.6 * *$ & 36.9 & $\ldots$ & $3.2 * *$ & $\ldots$ & $\ldots$ & $28.9 * * *$ & 46.3 & 16.5 & $\ldots$ & $8.2 * *$ & & $\ldots$ \\
\hline IND & $40.9 * * *$ & $22.5 * *$ & 35.4 & $\ldots$ & $\ldots$ & 1.1 & $\ldots$ & 5.3 & $51.0 *$ & 15.7 & $\ldots$ & $\ldots$ & $28.0 * * *$ & $\ldots$ \\
\hline HD & $35.1 * * *$ & $26.8 * *$ & 36.9 & $\ldots$ & $\ldots$ & $\ldots$ & 1.1 & 6.1 & $53.4 * *$ & 16.5 & $\ldots$ & $\ldots$ & $\ldots$ & $24.0 * * *$ \\
\hline RTA + RKI & $36.6 * * *$ & 18.5 & 36.9 & $4.7 * *$ & $3.2 * *$ & $\ldots$ & $\ldots$ & $31.5 * * *$ & 34.0 & 16.5 & $9.9 * *$ & $8.2 * *$ & $\ldots$ & $\ldots$ \\
\hline RTA + IND & $42.6 * * *$ & 12.6 & 35.4 & $8.2 * * *$ & 1.1 & $\ldots$ & $\ldots$ & 4.4 & 30.2 & 15.7 & $21.8 * * *$ & $\ldots$ & $28.0 * * *$ & $\ldots$ \\
\hline RTA + HD & $35.8 * * *$ & 18.4 & 36.9 & $7.9 * * *$ & $\ldots$ & $\ldots$ & 0.9 & $8.9 * *$ & 33.9 & 16.5 & $18.0 * * *$ & $\ldots$ & $\ldots$ & $22.8 * * *$ \\
\hline RKI + IND & $40.7 * * *$ & 19.3 & 35.4 & $\ldots$ & $3.4 * *$ & 1.1 & $\ldots$ & 4.7 & 43.8 & 15.7 & $\ldots$ & $8.1 * *$ & $28.0 * * *$ & $\ldots$ \\
\hline RKI + HD & $34.5 * * *$ & $24.5 *$ & 36.9 & $\ldots$ & $3.2 * *$ & $\ldots$ & 0.8 & $8.8 * *$ & 46.1 & 16.5 & $\ldots$ & $8.2 * *$ & $\ldots$ & $20.4 * * *$ \\
\hline IND + HD & $40.9 * * *$ & $22.3 *$ & 35.4 & $\ldots$ & $\ldots$ & 1.1 & 0.3 & 0.5 & $50.7 *$ & 15.7 & $\ldots$ & $\ldots$ & $28.0 * * *$ & $5.1 *$ \\
\hline RTA + RKI + IND & 42.5 *** & 12.7 & 35.4 & $4.8 * *$ & $3.4 * *$ & 1.1 & $\ldots$ & 4.4 & 30.2 & 15.7 & $13.6 * * *$ & $8.1 * *$ & $28.0 * * *$ & $\ldots$ \\
\hline RTA + RKI + HD & $35.9 * * *$ & 18.3 & 36.9 & $4.7 * *$ & $3.2 * *$ & $\ldots$ & 0.9 & $8.7 * *$ & 33.9 & 16.5 & $9.9 * *$ & $8.2 * *$ & $\ldots$ & $22.8 * * *$ \\
\hline RTA + IND + HD & $42.5 * * *$ & 12.6 & 35.4 & $8.2 * * *$ & $\ldots$ & 1.1 & 0.1 & 1.1 & 30.2 & 15.7 & $21.8 * * *$ & $\ldots$ & $28.0 * * *$ & 3.4 \\
\hline RKI + IND + HD & $40.7 * * *$ & 19.3 & 35.4 & $\ldots$ & $3.2 * *$ & 1.1 & 0.3 & 1.4 & 43.6 & 15.6 & $\ldots$ & $8.1 * *$ & $28.0 * * *$ & 3.2 \\
\hline
\end{tabular}

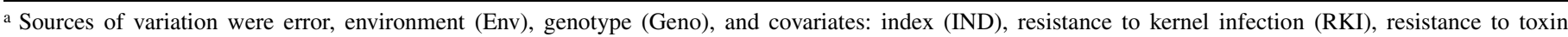
accumulation (RTA), and heading date (HD). Analyses were conducted using data from all four environments and using data from two environments (Ohio 2006 and Maryland 2008) that were independent of the two environments used to estimate RKI and RTA; * **, and *** indicate significant effects at $P$ levels $<0.05$, 0.01 , and 0.001 , respectively.

slopes from regressing DON on IND: when the slope is zero, all predicted values would be the mean DON of the environment, and we already established significant genetic effects on deviations from the mean for this set of genotypes (Table 6). The correlations of the regression residuals with deviations of observed DON values from their respective environment mean was 0.93. Similar results were obtained when using data from just the two environments with significant regressions.

ANOVAs for DON was conducted including the main effect of genotype and environment and single or multiple covariates. The covariates were IND, HD, RKI, and RTA. The use of DON data (versus residuals from regressing DON on IND) also allowed us to test IND as a covariate; that would not be possible with an analysis of the residuals as the linear effect of IND on DON was already modeled to obtain the residuals. The inclusion of covariates in the model affected the distribution of SoS in the analysis of DON when using data from either all four environments or just the two environments that were independent of the environments sampled in the RKI and RTA analyses (Table 9). All covariates were significant individually, except for HD in the analysis of all environments. Individually, using RTA as a covariate accounted for the greatest percentage of the total SoS. The inclusion of single or multiple covariates did not affect the error SoS in either analysis and, thus, the covariates modeled genotype or environment effects.

Including RTA as a covariate always reduced the genotype SoS (Table 9). The main effect of genotype on DON was significant without covariates but was always nonsignificant when RTA was 
included as a covariate either singularly or in combination with other covariates. The use of RKI as a covariate also reduced genotype $\mathrm{SoS}$ in both analyses, especially in the analyses of data from just two environments where inclusion of RKI as a covariate resulted in nonsignificant genotype effects on DON. A combination of RKI and IND, and also RKI, IND, and HD, reduced genotype SoS in both analyses (Table 9). Neither IND of HD greatly affected genotype SoS.

Including RTA or RKI as covariates did not affect environment SoS. Including either IND and HD as covariates greatly reduced environment SoS in the analysis of data from two environments (Table 9). These two environments (Ohio in 2006 and Maryland in 2008) differed greatly for average IND and DON (Table 7); including IND as a covariate apparently modeled much of the environment effect for DON for these two environments. A similar trend was noted when HD was used as a covariate in these environments: IND and HD were correlated in the Maryland environment.

The most important covariate for modeling DON was RTA. It was significant in all analyses and always produced a large decrease in genotype SoS with no affect on environment SoS. Including RTA as a covariate reduced genotype SoS by $32 \%$ in the analysis of four environments and by $44 \%$ in the analysis of two environments. Similar results were seen when RTA was combined with other covariates. The correlation RTA and mean DON over four environments $(r=0.58)$ or from two environments $(r=$ 0.61 ) was significant while the correlation of IND, HD, and RKI with DON was not significant (results not shown).

\section{DISCUSSION}

Significant genotype effects on all FHB traits were noted despite the lack of pedigree diversity and selection of lines with moderate resistance. This concurs with previous reports on related soft winter wheat populations (40). Genotype-set interactions were significant in the RKI and RTA study for DON and FB (Table 2). These interactions are common in FHB field studies. Due to bulking grains samples over replications and the method used to estimate RKI and RTA parameters, we could not test the significance of the genotype-set interaction for the other analyses (Tables 5, 6, and 8). Inspection of relative magnitude of the error SoS, the ability to detect significant genetic effects, and the relatively high heritability of IND and DON suggest that the interactions did not mask important genetic trends in the data.

Genetic effects for RKI and RTA. The level of DON and FB in the grain increased as number of SS increased (Table 3 ). The significance of the genotype-SS interaction (Table 2) indicates that this response was not equal among all genotypes. There were significant genetic effects for both RKI and RTA (Table 5). Some genotypes had a consistent response in all four sets, and genotypes with consistent resistant or susceptible responses were identified for RKI and RTA (Table 4). The different levels of RKI and RTA between these genotypes would have an important impact on controlling DON levels in grain. Genotypes 2-1, 6-1, 6-2, and 9-1 consistently showed little to no increase in FB levels in their grain (Fig. 1) despite increasing the number of SS from 0 to 3 (e.g., raising IND from 0 to $17.7 \%$ for a 17 -spikelet spike). In contrast, genotypes 1-3, 2-2, 2-3, and 10-2 showed increases of 1.1 to 9.3 units of FB for each infected spikelet.

The difference among genotypes was more striking for RTA than for RKI and may have a greater impact on controlling DON levels in grain. The average $r^{2}$ values for RTA regressions were 0.39 versus 0.24 for RKI (Table 4). Genotypes 1-3, 9-1, 13-1, and 13-2 consistently showed negligible increases in DON despite experiencing $\mathrm{FB}$ levels in their grain equal to that of more susceptible genotypes such as 2-4, 3-3, and 8-2 (Fig. 2). For example, the slope from regressing DON on FB for the resistant genotype $1-3$ in the four sets was $-1.4,-2.2,0.0$, and -0.8 while the slopes for the susceptible genotype $2-4$ were $0.9,6.1,4.5$, and 18.5. The raw values of FB levels for genotype $1-3$ were 0.2 to $22.9 \mu \mathrm{g}$ whereas the observed DON values were all $<7.3 \mathrm{ppm}$. In contrast, genotype 2-4 had approximately half the range of raw FB values ( 0.3 to $9.4 \mu \mathrm{g})$ but observed DON values of 2.7 to 33 ppm. Our finding of little to no increase in DON with increasing FB in the grain of some genotypes is in contrast to reports of significant positive regressions of DON on FB using grain samples collected from single cultivars (7) or random field samples $(11,45)$.

Association of RKI and RTA with DON and IND data from whole plots. Our second objective was to determine whether variation for RKI and RTA could explain why some genotypes with moderate resistance to FHB based on IND produce grain with high DON levels. We evaluated IND and DON in four environments for 75 genotypes, including the 32 used in the RKI and RTA study. All RILs had average IND values less than the moderate check, though 21 RILs had numerically higher DON levels than the susceptible check. The association of DON with IND was not very strong (Table 7). The lack of a meaningful association of IND and DON is common among genotypes with moderate resistance $(27,31-33,39)$. It also shows that the genetic variation for DON in this set of lines is not associated with variation for type I or II resistance assessed with IND.

To determine whether RKI or RTA was associated with DON in these moderately resistant genotypes, we used RKI, RTA, IND, and $\mathrm{HD}$ as covariates in the analysis of DON from grain harvested from whole plots. All DON data in this analysis were obtained from grain samples that were independent of those used to estimate RKI and RTA. We did one analysis using data from all four environments and a second analysis using data from the two environments that were not used to estimate RKI or RTA. No covariate affected the error SoS, indicating that the covariates modeled environment or genotype effects. All four covariates were significant (Table 9) though they had different effects on environment and genotype SoS. The use of IND and HD as covariates impacted environment SoS but had little effect on genotype SoS. The use of RKI as a covariate had little impact on environment SoS and caused a modest reduction in genotype SoS. The use of RTA as a covariate reduced the genotype SoS by 32 to $44 \%$ in the two analyses but had little impact on environment SoS. In addition, RTA was correlated to DON in both data sets though RKI and IND were not. This indicates that RTA explains a substantial amount of the genetic variation for DON levels among genotypes with moderate type I and/or type II resistance.

Conclusions. There are multiple lines of evidence for genetic variation for RKI and RTA: the significant genotype-SS interaction (Table 2), the significant genetic differences for RKI and RTA slopes (Table 5), and the reduction of genotype SoS when using RKI and RTA as covariates in the DON analysis (Table 9). To our knowledge, this is the first report to show that wheat genotypes repeatedly vary for RKI and RTA using accurate assessment of infection. Jansen et al. (16) reported that $F$. graminearum penetrated the caryopsis of 'Chevron' barley more slowly than the caryopsis of other barley cultivars, suggesting variation for RKI in barley. Others $(27,29,41)$ have shown that some genotypes have less DON and lower FDK levels than predicted based on type I or II infection but did not repeat these deviations or measure actual infection.

In this study, RKI and RTA were correlated $(r=0.58)$ but neither was significantly correlated to IND, which is a function of type I and II resistance. The low correlation with IND may be due, in part, to the limited range of IND values because the study used genotypes selected for moderate to low IND values. Still, the lack of significant correlations with IND suggests that RKI and RTA in the grain are independent of type I and II resistance in this population, and all mechanisms could be combined to produce low DON levels. 
The correlation of RKI and RTA suggests the possibility of a common mechanism between them. It is well documented that $F$. graminearum needs to produce DON to spread through the vegetative tissue of a wheat spike $(1,8,9,13,16,21,25)$. Although it seems logical that DON accumulation facilitates kernel infection, the role of DON in kernel infection is not well documented. Jansen et al. (16) found that $F$. graminearum strains with and without the ability to produce DON had equal ability to infect wheat and barley caryopsis, suggesting that DON production may not be very important to kernel infection. Ilgen et al. (15), however, showed that the F. graminearum Tri5 gene for DON synthesis is induced during kernel infection, suggesting a role for DON in kernel infection.

Selecting for low IND, a function of type I and II resistance, reduces DON although, once IND values get below a moderate level, then IND is only weakly associated with DON (33). Thus, once those IND levels are reached, selection for even lower IND will likely have little impact on DON. At that point, genetic variation for RKI and RTA would become important to control DON levels, with RTA being the main factor that should be used in selection for low DON.

Selection for RKI and RTA would represent a new approach to breeding for low DON but also presents challenges. The method we present in this article is not practical for assessing RTA or RKI in large breeding populations or even mapping populations due to the extensive sampling of spikes with different levels of symptoms from each genotype. Other methods could be used to develop a series of grain samples with different infection levels per genotype. However, these would also seem to be laborious and there remains the high cost of doing quantitative PCR on all sample to assess FB.

A practical approach could be to first select for genotypes with low IND values and then select for genotypes with low FDK and DON, because RTA and DON were correlated in this study: selecting for low DON should indirectly select for good RTA. Unfortunately, DON has low heritability when assessed in few environments, and indirect selection for RTA using a trait with low heritability would likely have little impact on RTA. Thus, to really improve RTA, one will need to quantify FB among the selected genotypes and then select genotypes with lower DON than expected based on FB. An assay for specific $F$. graminearum proteins may be an alternative to costly quantitative PCR. This discussion points out the need to develop more efficient assays for RTA, likely based on inexpensive biochemical assays or from identifying and validating expression of candidate genes such as those reported by Boddu et al. (2,3), Boutigny et al. (4), Lemmens et al. (22), Schweiger et al. (37), Steiner et al. (43), and Walter et al. (46). Additional germplasm can be screened for RTA, though the slopes we report are close to zero; therefore, it is not clear how much more genetic variation is needed to optimize RTA.

\section{ACKNOWLEDGMENTS}

Salaries and research support were provided by state and federal funds to the Ohio Agricultural Research and Development Center. This investigation is based upon work supported, in part, by the United States Department of Agriculture (agreement number 59-0206-9-086). This is a cooperative project with the U.S. Wheat \& Barley Scab Initiative.

\section{LITERATURE CITED}

1. Bai, G. H., Desjardins, A. E., and Plattner, R. D. 2001. Deoxynivalenolnonproducing Fusarium graminearum causes initial infection, but does not cause disease spread in wheat spikes. Mycopathologia 153:91-98.

2. Boddu, J., Choo, S., Kruger, W., and Muehlbauer, G. 2006. Transcriptome analysis of the barley-Fusarium graminearum interaction. Mol. PlantMicrobe Interact. 19:407-417.

3. Boddu, J., Choo, S., and Muehlbauer, G. 2007. Transcriptome analysis of trichothecene-induced gene expression in barley. Mol. Plant-Microbe Interact. 20:1364-1375.
4. Boutigny, A.-L., Richard-Forget, F., and Barreau, C. 2008. Natural mechanisms for cereal resistance to the accumulation of trichothecenes. Eur. J. Plant Pathol. 121:411-423.

5. Buerstmayr, H., Ban, T., and Anderson, J. A. 2009 QTL mapping and marker-assisted selection for Fusarium head blight resistance in wheat: a review. Plant Breed. 128:1-26.

6. Cowger C., and Arrellano, C. 2010. Plump kernels with high deoxynivalenol linked to late Gibberella zeae infection and marginal disease conditions in winter wheat. Phytopathology 100:719-28.

7. Demeke, T., Gaba, D., Patrick, S. K., Seifert, K. A., Levesque, C. A., Chapados, J., Clear, R. M., Grafenhan, T., Ratnayaka, I., and Phan, A. 2010. Development of a specific TaqMan real-time PCR assay for quantification of Fusarium graminearum clade 7 and comparison of fungal biomass determined by PCR with deoxynivalenol content in wheat and barley. Int. J. Food Microbiol. 141:45-50.

8. Desjardins, A. E., Proctor, R. H., Bai, G., McCormick, S. P., Shaner, G., Buechley, G., and Hohn, T. M. 1996. Reduced virulence of trichothecenenonproducing mutants of Gibberella zeae in wheat field tests. Mol. PlantMicrobe Interact. 9:775-781.

9. Eudes, F., Comeau, A., Rioux, S., and Collins, J. 2001. Impact of trichothecenes on Fusarium head blight (Fusarium graminearum) development in spring wheat (Triticum aeastivum). Can. J. Plant Pathol. 23:318-322.

10. Farve, L., Verdal-Bonnin, M., Pinson-Gadais, L., Roumet, P., Barreau, C., and Richard-Forget, F. 2004. Does biochemical composition of durum wheat kernels influence the trichothecene B (TCT B) contamination level? In: 2nd Int. Symp. Fusarium Head Blight, Orlando, FL.

11. Fredlund, E., Gidlund, A., Olsen, M., Börjesson, T., Spliid, N. H. H., and Simonsson, M. 2008. Method evaluation of Fusarium DNA extraction from mycelia and wheat for down-stream real-time PCR quantification and correlation to mycotoxin levels. J. Microbiol. Methods 73:33-40.

12. Fuentes, R. G., Mickelson, H. R., Busch, R. H., Dill-Macky, R., Evans, C. K., Thompson, W. G., Wiersma, J. V., Xie, W., Dong, Y., and Anderson, J. A. 2005. Resource allocation and cultivar stability in breeding for Fusarium head blight resistance in spring wheat. Crop Sci. 45:1965-1972.

13. Goswami, R. S., and Kistler, H. C. 2005. Pathogenicity and in planta mycotoxin accumulation among members of the Fusarium graminearum species complex on wheat and rice. Phytopathology 95:1397-1404.

14. Horsley, R. D., Franckowiak, J. D., Schwarz, P. B., Zhang, B., Green, M. J., Steffenson, B. J., Maier, C., Schmierer, D., Urrea, C. A., and Kudrna, D. 2006. Identification of QTLs associated with Fusarium head blight resistance in barley accession CIho 4196. Crop Sci. 46:145-156.

15. Ilgen, P., Hadeler, B., Maier, F. J., and Schäfer, W. 2009. Developing kernel and rachis node induce the trichothecene pathway of Fusarium graminearum during wheat head infection. Mol. Plant-Microbe Interact. 22:899-908.

16. Jansen, J. D., von Wettstein, X., Shafer, W., Kogel, H.-H., Felk, A., and Maier, F. J. 2005. Infection patterns in barley and wheat spikes inoculated with wild type and tirchodiene synthase gene disrupted Fusarium graminearum. Proc. Natl. Acad. Sci. USA 102:16892-16897.

17. Jiang, G., Ward, R. W., Shi, J., and Dong, Y. 2007. QTL analysis of resistance to Fusarium head blight in the novel wheat germplasm CJ 9306. II. resistance to deoxynivalenol accumulation and grain yield loss. Theor. Appl. Genet.115:1043-1052.

18. Kang, J., Clark, A., Van Sanford, D., Griffey, C., Brown-Guedira, G. L., Dong, Y., and Costa, J. 2011. Exotic scab resistance quantitative trait loci (QTL) effects on soft red winter wheat. Crop Sci. 51:924-933.

19. Knapp, S. J., Stroup, W. W., and Ross, W. M. 1985. Exact confidence intervals for heritability on a progeny mean basis. Crop Sci. 25:192-194.

20. Lamb, K. E., Gonzalez-Hernandez, J., Zhang, B., Green, M., Neate, S. M., Schwarz, P. B., and Horsley, R. D. 2009. Identification of QTL conferring resistance to Fusarium head blight resistance in the breeding line C93-3230-24. Crop Sci. 49:1675-1680.

21. Langevin, F., Eudes, F., and Comeau, A. 2004. Effect of trichothecenes produced by Fusarium graminearum during Fusarium head blight development in six cereal species. Eur. J. Plant Pathol. 110:735-746.

22. Lemmens, M., Scholz, U., Berthiller, F., Dall'Asta, C., Kouynik, A., Schuhmacher, R., Adam, G., Buerstmayr, H., Mesterházy, A., Krska, R., and Ruckenbauer, P. 2005. The ability to detoxify the mycotoxin deoxynivalenol colocalizes with a major quantitative trait locus for Fusarium head blight resistance in wheat. Mol. Plant-Microbe Interact. 18:1318-1324

23. Loffler, M., Schon, C.-C., and Miedaner, T. 2009. Revealing the genetic architecture of FHB resistance in hexaploid wheat (Triticum aestivum L.) by QTL meta-analysis. Mol. Breed. 23:473-488.

24. Ma, H. X., Chen, H. G., Cai, Z. X., Lu, W. Z., Zhang, K. M., Gao, L., and Bai, G. H. 2006. Quantitative trait loci for resistance to Fusarium head blight and deoxynivalenol accumulation in Wangshuibai wheat under field conditions. Plant Pathol. 55:739-745.

25. Maier, F. J., Miedaner, T., Hadeler, B., Felk, A., Salomon, S., Lemmens, M., Kassner, H., and Shafer, W. 2006. Involvement of trichothecenes in 
fusarioses of wheat, barley, and maize evaluated by gene disruption of the trichodiene synthase (Tri5) gene in three field isolates of different chemotype and virulence. Mol. Plant Pathol. 7:449-461.

26. Marasas, W. F, Nelson, P. E., and Toussoun, T. A. 1984. Toxigenic Fusarium Species: Identity and Mucotoxicology. The Pennsylvania State University Press, University Park.

27. Mesterházy, A. 1995. Types and components of resistance to Fusarium head blight of wheat. Plant Breed. 114:377-386.

28. Mesterházy, A. 2002. Role of deoxynivalenol in aggressiveness of Fusarium graminearum and F. culmorum and in resistance to Fusarium head blight. Eur. J. Plant Pathol. 108:675-684.

29. Mesterházy, Á., Bartók, T., Mirocha, C. G., and Komoróczy, R. 1998. Nature of wheat resistance to Fusarium head blight and the role of deoxynivalenol for breeding. Plant Breed. 118:97-110.

30. Miller, J. D., and Arnison, P. 1986. Degradation of deoxynivalenol by suspension cultures of the Fusarium head blight resistant wheat cultivar Frontana. Can. J. Plant Pathol. 8:147-150.

31. Paul, P. A., Lipps, P. E., and Madden, L. 2005. Relationship between visual estimates of Fusarium head blight intensity and deoxynivalenol accumulation in harvested wheat grain: A meta-analysis. Phytopathology 95:1225-1236.

32. Paul, P. A., El-Allaf, S. M., Lipps, P. E., and Madden, L. V. 2005. Relationships between incidence and severity of Fusarium head blight on winter wheat in Ohio. Phytopathology 95:1049-1060.

33. Paul, P. A., Lipps, P. E., and Madden, L. V. 2006. Meta-analysis of regression coefficients for the relationship between Fusarium head blight and deoxynivalenol content of wheat. Phytopathology 96:951-961.

34. Pitt, J. I., Basilico, J. C., Abarca, M. L., and Lopez, C. 2000. Mycotoxins and toxigenic fungi. Med. Mycol. 38(Suppl. 1):41-46.

35. Reid, L., Zhu, X., Savard, M. E., Sinha, R. C., and Vigier, B. 2000. Preharvest accumulation of deoxynivalenol in sweet corn ears inoculated with Fusarium graminearum. Food Addit. Contam. 8:689-701.

36. SAS Institute Inc. 2002-2004. SAS 9.1.3, SAS Institute Inc., Cary, NC.

37. Schweiger, W., Lemmens, M., Muehlbauer, G. J., Adam, G., Berthiller, F., Boddu, J., Shin, S., and Poppenberger, B. 2010. Validation of a candidate deoxynivalenol-inactivating UDP-glucosyltransferase from barley by heterologous expression in yeast. Mol. Plant-Microbe Interact. 23:977-986.

38. Semagn, K., Maroy, A. G., Tarkegne, Y., Skinnes, H., and Bjornstad, A.
2007. Quantitative trait loci controlling Fusarium head blight resistance and low deoxynivalenol content in hexaploid wheat population from 'arina' and NK93604. Crop Sci. 47:294-303.

39. Smith, K. P., Evans, C. K., Dill-Macky, R., Gustus, C., Xie, W., and Dong, Y. 2004. Host genetic effects on deoxynivalenol accumulation in Fusarium head blight of barley. Phytopathology 94:766-771.

40. Sneller, C., Paul, P. A., and Guttieri, M. 2010. Characterization of resistance to Fusarium head blight in an Eastern US soft red winter wheat population. Crop Sci. 50:123-133.

41. Snijders, C. H. A, and Perkowski, J. 1990. Effect of head blight caused by Fusarium culmorum on toxin content and weight of wheat kernels. Phytopathology 80:133-140.

42. Somers, D. J., Thomas, J., DePauw, R., Fox, S., Humpherys, G. and Fedak, G. 2005. Assembling complex genotypes to resist Fusarium in wheat (Triticum aestivum L.). Theor. Appl. Genet. 111:1623-1631.

43. Steiner, B., Kurz, H., Lemmens, M., and Buerstmayr, H. 2009. Differential genes expression of related wheat lines with contrasting levels of head blight resistance after Fusarium graminearum inoculation. Theor. Appl. Genet. 118:753-764.

44. Voigt, C. A., Shafer, W., and Salomon, S. 2005. A secreted lipase of $F u$ sarium graminearum is a virulence factor required for infection of cereals. Plant J. 42:364-375.

45. Waalwijk, C., van der Heide, R., de Vries, I., van der Lee, L., Schoen, C., Costrel-de Corainville, G., Häuser-Hahn, I., Kastelein, P., Köhl, J., Lonnet, P., Demarquet, T., and Kema, G. H. J. 2004. Quantitative detection of Fusarium species in wheat using TaqMan. Eur. J. Plant Pathol. 110:481-494.

46. Walter, S. J., Brennan, M., Arunachalam, C., Ansari, K., Hu, X., Khan, M., Trognitz, F., Trognitz, B., Leonard, G., Egan, D., and Doohan, F. M. 2008. Components of the gene network associated with genotypedependent response of wheat to Fusarium mycotoxin deoxynivalenol. Funct. Integr. Genomics 8:421-427.

47. Yao, Q., Liu, Z., and Zeng, Y. 1996. Detoxification of deoxynivalenol by scab resistant wheat and the bioactivities of the products. Acta Mycol. Sin. 15:59-64

48. Yu, J. B., Dong, Y. H., Kolb, F. L., Bai, G. H., and Zhou, W. C. 2008. Quantitative trait loci for Fusarium head blight resistance in a recombinant inbred population of Wangshuibai/Wheaton. Phytopathology 98:87-94. 